\title{
Impact of Synchrophasor Measurement Types and Uncertainties on the Accuracy of Distribution System Linear State Estimators
}

\author{
Lorenzo Zanni, Daniele Colangelo, Rachid Cherkaoui and Mario Paolone \\ École Polytechnique Fédérale de Lausanne (EPFL) \\ Lausanne, Switzerland \\ lorenzo.zanni@epfl.ch
}

\begin{abstract}
The paper aims at assessing the effects of combined voltage and/or current synchrophasor measurements, and their associated uncertainties, on the accuracy of state estimators adopted in distribution systems. Such an assessment is first carried out with respect to a generic transmission line with the purpose of determining the combination of voltage and/or current synchrophasor measurements that provides the best accuracy of the estimated quantities. A comprehensive analysis on the impact of different measurement uncertainties and operating conditions is included for this specific case. In order to derive general conclusions, the study is then extended to a distribution system composed of the IEEE 13-bus test feeder. For this case, we perform an a-posteriori assessment of the probability distributions of the estimation errors by using a discrete Kalman filter state estimator fed with noisy voltage and/or injected current synchrophasor measurements.
\end{abstract}

Index Terms - Synchrophasors, distribution systems, state estimation, accuracy.

\section{INTRODUCTION}

The increasing penetration of distributed energy resources (DERs) in distribution systems might require specific control schemes based on the quasi real-time knowledge of the network state with both high accuracy and refresh-rate. In order to achieve this objective, a new category of real-time state estimators based on synchrophasor measurements has been proposed in the recent literature (e.g., [1]-[3]). Nowadays, phasor measurement units (PMUs) are able to acquire accurate synchrophasors streamed at some tens of frames-per-second [4]. Within the context of real-time monitoring of active distribution networks, advanced PMUs exhibiting high accuracy and resilience against fast transients and distorted waveforms have been developed [5]-[7]. The availability of this kind of measurements enables the formulation of dedicated state estimation (SE) processes that are generally linear (e.g., [3], [8]-[11]).

The type, placement and accuracy of the measurement devices have a significant impact on the SE accuracy. Consequently, a proper selection of these characteristics is required in order to analyze the SE performances. In this respect, a large part of the literature focuses on the definition of methods for the optimal PMU placement with the aim of providing the minimal number of PMU installations to ensure the full observability of the power system. In [12], Manousakis et. al have presented a taxonomy of the works devoted to this subject. Other papers have proposed specific methods to identify the most appropriate measurement location in order to improve the quality of SE processes [13][17]. In [13], Li has proved how real-time measurements improve the SE accuracy, whereas the use of pseudomeasurements can degrade the SE results. A Monte Carlobased approach that seeks the buses with the largest errors of voltage and phase estimates as a potential location for meter placement is presented in [14]. In [15], Caro et al. propose a heuristic method to optimally locate a predefined number of PMUs throughout an observable system in order to maximize the SE accuracy. In [16], Liu et. al propose an optimization process able to determine the optimal meter placement with trade-offs among SE accuracy, number of smart meters and number of PMUs in addition to pseudo-measurements. The impact of different types of measurements (such as powers provided by smart meters, synchrophasors, and pseudomeasurements), measurement accuracies, and operating conditions on SE results is analyzed in [17].

In general, these works have not assessed the combined effects of synchrophasor-only measurement types and uncertainties on the performance of a SE process: this paper investigates this specific aspect. Section II presents a preliminary study carried out by considering a simple twoports equivalent of a transmission line. We evaluate the impact on the estimated quantities of different configurations of voltage and/or current synchrophasor measurements, measurement uncertainties, line types (overhead or cable), as well as operating conditions. Then, in Section III the study is extended to the IEEE 13-bus distribution test feeder in order to generalize the findings of Section II. The metric used is the accuracy of a three-phase state estimator based on the discrete Kalman filter (DKF). 


\section{ASSESSMENT PROCEDURE FOR THE CASE OF A GENERIC TRANSMISSION LINE}

\section{A. Description of the physical system}

This section introduces the procedure for assessing the influence of different measurement configurations on the accuracy of the estimated quantities. It makes use of a twoports equivalent of a generic transmission line. The latter is represented by the well-known single-phase ${ }^{1}$ PI circuit shown in Fig. 1. The longitudinal $\underline{Y}_{L}$ and transverse $\underline{Y}_{T}$ admittances of the line are supposed to be known and defined as (the under bar identifies complex quantities and $j$ is the imaginary unit):

$$
\underline{Y}_{L}=[(r+j x) \cdot L]^{-1} \quad, \quad \underline{Y}_{T}=(g+j b) / 2 \cdot L,
$$

where $r$ and $x$ are respectively the per-unit length longitudinal resistance and reactance, $g$ and $b$ are respectively the per-unit length transverse conductance and susceptance, and $L$ is the line length. The circuit of Fig. 1 is henceforth represented by the parameters of the equivalent $A B C D$ auxiliary matrix.

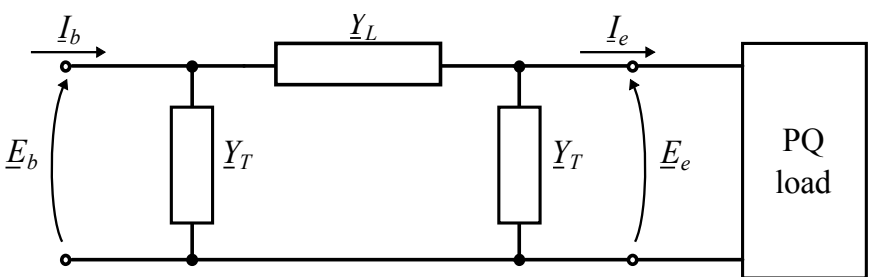

Fig. 1. Two-ports equivalent of a transmission line represented by the singlephase PI circuit. $\underline{E}_{b}$ and $\underline{E}_{e}$ are the voltage phasors at the beginning and at the end of the line, while $\underline{I}_{b}$ and $\underline{I}_{e}$ are the current phasors.

With respect to the system of Fig. 1, the six possible measurement configurations are:
a. $\left(\underline{E}_{b}^{M}, \underline{E}_{e}^{M}\right)$
b. $\left(\underline{I}_{b}^{M}, \underline{I}_{e}^{M}\right)$
c. $\left(\underline{E}_{e}^{M}, \underline{I}_{e}^{M}\right)$
d. $\left(\underline{E}_{b}^{M}, \underline{I}_{b}^{M}\right)$
e. $\left(\underline{E}_{e}^{M}, \underline{I}_{b}^{M}\right)$
f. $\left(\underline{E}_{b}^{M}, \underline{I}_{e}^{M}\right)$,

where $\underline{E}_{b}$ and $\underline{E}_{e}$ are respectively the voltage phasors at the beginning and at the end of the line, $\underline{I}_{b}$ and $\underline{I}_{e}$ are the current phasors, and the apex $M$ stands for 'measured quantity'. Since for transmission lines the two-ports equivalent is reciprocal, configurations $c, d, e$ and $f$ are interchangeable. Thus, only configuration $c$ is used in the analysis. The computed quantities (indicated by the apex $C$ in (3)-(5)) are derived by means of the auxiliary matrices that correspond to the three considered measurement configurations:

a. $\quad\left[\begin{array}{c}\underline{I}_{b}^{C} \\ \underline{I}_{e}^{C}\end{array}\right]=\left[\begin{array}{cc}\underline{Y}_{T}+\underline{Y}_{L} & -\underline{Y}_{L} \\ \underline{Y}_{L} & -\left(\underline{Y}_{T}+\underline{Y}_{L}\right)\end{array}\right]\left[\begin{array}{l}\underline{E}_{b}^{M} \\ \underline{E}_{e}^{M}\end{array}\right]$,
b. $\quad\left[\begin{array}{l}\underline{E}_{b}^{C} \\ \underline{E}_{e}^{C}\end{array}\right]=\left[\begin{array}{cc}\frac{\underline{Y}_{L}+\underline{Y}_{T}}{\underline{Y}_{T}\left(2 \underline{Y}_{L}+\underline{Y}_{T}\right)} & \frac{-\underline{Y}_{L}}{\underline{Y}_{T}\left(2 \underline{Y}_{L}+\underline{Y}_{T}\right)} \\ \frac{\underline{Y}_{L}}{\underline{Y}_{T}\left(2 \underline{Y}_{L}+\underline{Y}_{T}\right)} & \frac{-\left(\underline{Y}_{L}+\underline{Y}_{T}\right)}{\underline{Y}_{T}\left(2 \underline{Y}_{L}+\underline{Y}_{T}\right)}\end{array}\right]\left[\begin{array}{l}\underline{I}_{b}^{M} \\ \underline{I}_{e}^{M}\end{array}\right]$,

\footnotetext{
${ }^{1}$ A single-phase analysis is sufficient to derive preliminary conclusions that will be verified in Section III by using a three-phase distribution system.
}

c. $\quad\left[\begin{array}{c}\underline{E}_{b}^{C} \\ \underline{I}_{b}^{C}\end{array}\right]=\left[\begin{array}{cc}\underline{Y}_{L}+\underline{Y}_{T} & \frac{1}{\underline{Y}_{L}} \\ \underline{Y}_{T}\left[2+\frac{\underline{Y}_{T}}{\underline{Y}_{L}}\right] & \frac{\underline{Y}_{L}+\underline{Y}_{T}}{\underline{Y}_{L}}\end{array}\right]\left[\begin{array}{l}\underline{E}_{e}^{M} \\ \underline{I}_{e}^{M}\end{array}\right]$.

\section{B. Description of the measurement model}

The uncertainty of the measurements is another parameter that, together with the selected measurement configuration, plays a crucial role on the evaluation of the accuracy of the computed quantities. In particular, we are interested in quantifying the influence of the magnitude and phase measurement errors separately. Therefore, the variation of the magnitude error, assuming a null phase error, allows evaluating the effect of the magnitude error and vice versa. As known, the performance of a PMU can be expressed in terms of total vector error (TVE) [4]. The maximum magnitude error $e r r_{m}$ (or phase error err $_{p}$ ) is calculated from the assumed TVE by considering a null phase (or magnitude) error:

$$
e r r_{m}=\left.f(T V E)\right|_{e r r_{p}=0}, e r r_{p}=\left.f(T V E)\right|_{e r r_{m}=0},
$$

Then, we simulate the measurements by adding to the true values of the quantities of (2) a randomly-generated noise ( $\Delta m$ for the magnitude and $\Delta p$ for the phase) assumed to be Gaussian, white and with a standard deviation (std) equal to $1 / 3$ of the maximum error in order to cover the $99.7 \%$ of the Gaussian distribution [18]:

$$
\begin{array}{ll}
\Delta m & N\left(0, \text { err }_{m} / 3\right), X_{m}^{M}=X_{m}^{T}+\Delta m, \\
\Delta p & N\left(0, \text { err }_{p} / 3\right), X_{p}^{M}=X_{p}^{T}+\Delta p
\end{array}
$$

where $X$ is a generic quantity of (2) and the apex $T$ stands for 'true quantity'.

\section{Description of the assessment procedure}

For a given scenario, the procedure used to assess the accuracy of the computed quantities as a function of the uncertainty of the measured quantities is the following:

1. A simple power flow is computed in order to determine the true state of the network shown in Fig. 1 by imposing the powers at the end of the line;

2. $\quad N$ sets of measurements are obtained by perturbing the true quantities inferred from step 1 with randomlygenerated Gaussian noise according to (7). The selected number of draws is equal to $10^{4}$ in order to infer statistical distributions that are numerically significant;

3. $\quad N$ sets of computed quantities are calculated by applying the auxiliary matrices of (3)-(5) to each set of measurements. Then, we calculate the errors as the difference between computed and true quantities;

4. The accuracy of the computed quantities is represented by the stds of the probability distributions of the errors calculated in step 3 . 


\section{Numerical example}

This section presents a numerical analysis of the influence of the three measurement configurations selected in Section II on the accuracy of the computed quantities. The analysis includes different measurement uncertainties and operating conditions. We consider characteristic parameters of typical overhead lines used in distribution systems (see Table I). The operating conditions shown in Table II refer to different powers absorbed by the load considering a power factor of 0.9. It is worth mentioning that the analysis has been carried out also by using underground cables and a power factor of 0.5: the same conclusions hold in both cases. For the sake of comparison among the various scenarios, the magnitude error is relative to the amplitude of the true quantity and the phase error is expressed in radians.

Fig. 2 refers to measurement configuration $a$ in which the voltage phasors are measured and the current phasors are calculated. It shows the separate influence of the magnitude and phase measurement errors (expressed in terms of TVE \%) on the accuracy of the magnitude and phase of $\underline{I}_{b}{ }^{C}$ (same results are obtained for $\underline{I}_{e}^{C}$ ). It can be seen that the operating condition highly influences the accuracy of the computed quantities. The case of very low power flow is the most challenging since the voltage magnitude variations, as well as the phase displacements at the line extremities are extremely small.

TABLE I

ELECTRICAL PARAMETERS OF THE LINE.

\begin{tabular}{|c|c|c|c|c|c|}
\hline Line type & $r[\Omega / \mathrm{km}]$ & $x[\Omega / \mathrm{km}]$ & $g[\mu \mathrm{S} / \mathrm{km}]$ & $b[\mu \mathrm{S} / \mathrm{km}]$ & $L[\mathrm{~km}]$ \\
\hline overhead & 0.268 & 0.346 & 0 & 3.36 & 5 \\
\hline cable & 0.0995 & 0.0971 & 0.285 & 94.87 & 0.5 \\
\hline
\end{tabular}

TABLE II

OPERATING CONDITIONS: APPARENT POWER.

\begin{tabular}{|c|c|c|c|c|}
\hline$S[\mathrm{kVA}]$ & 1 & 10 & 100 & 2000 \\
\hline
\end{tabular}
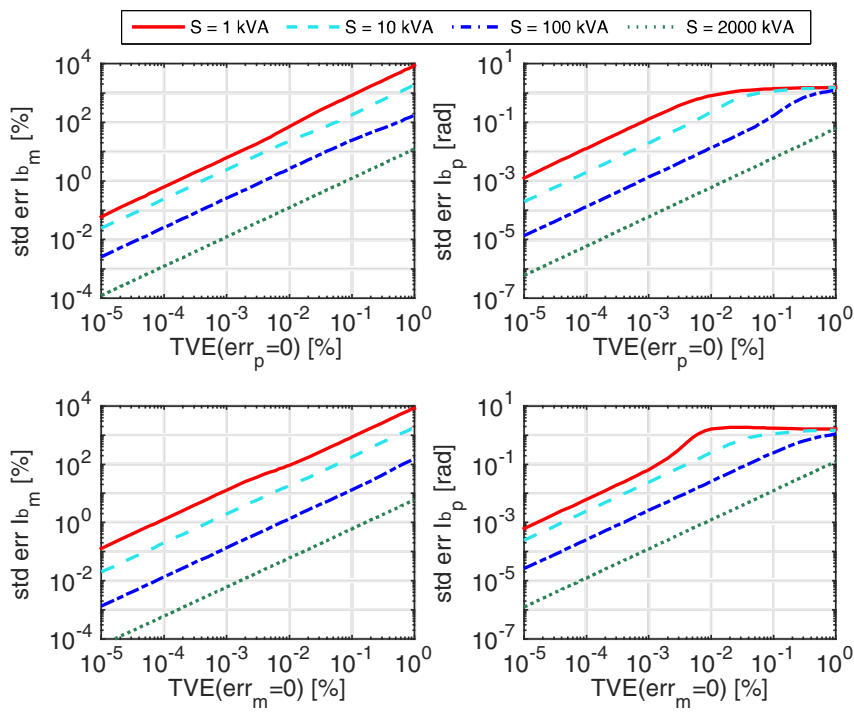

Fig. 2. Measurement configuration $a$. Accuracy of the magnitude and phase of $\underline{I}_{b}^{C}$ (same results are obtained for $\underline{I}_{e}{ }^{C}$ ) as a function of the uncertainty of the magnitude and phase of the voltage measurements expressed in TVE \%.
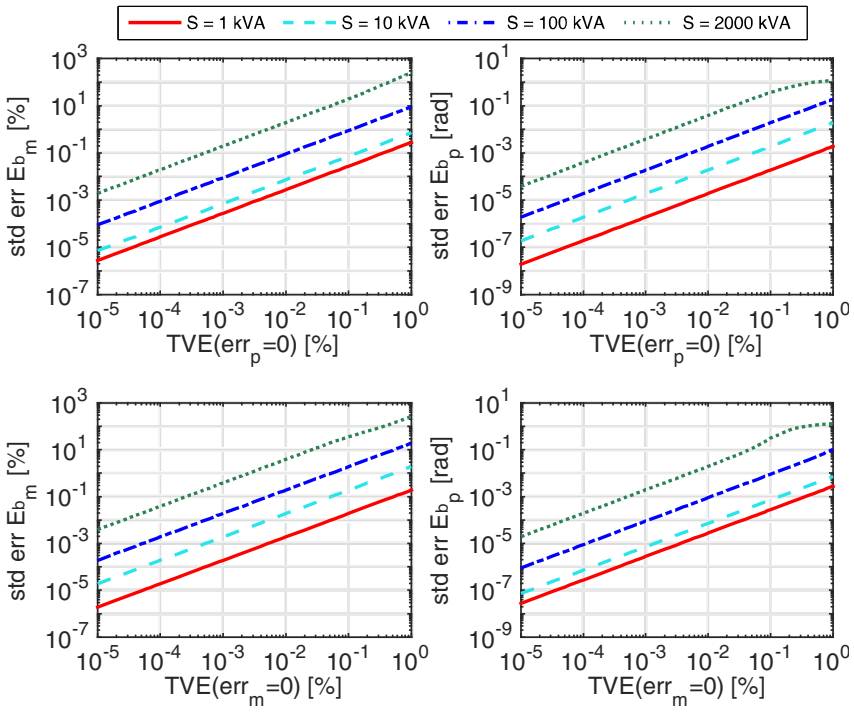

Fig. 3. Measurement configuration $b$. Accuracy of the magnitude and phase of $\underline{E}_{b}{ }^{C}$ (same results are obtained for $\underline{E}_{e}{ }^{C}$ ) as a function of the uncertainty of the magnitude and phase of the current measurements expressed in TVE $\%$.
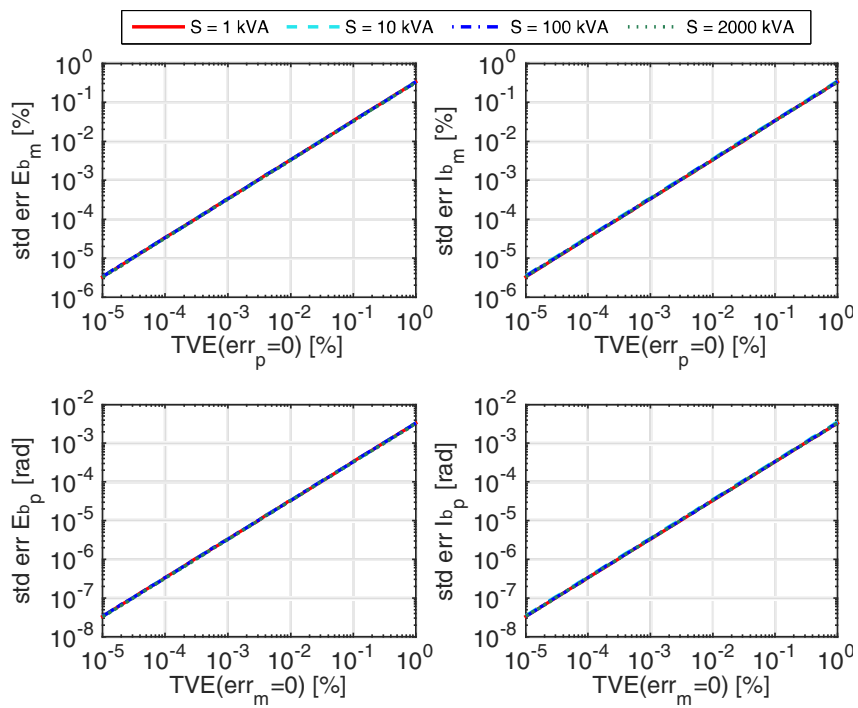

Fig. 4. Measurement configuration $c$. The two upper graphs show the accuracy of the magnitude of $\underline{E}_{b}{ }^{C}$ and $\underline{I}_{b}{ }^{C}$ as a function of the uncertainty of the magnitude of the $\underline{E}_{e}{ }^{M}$ and $\underline{I}_{e}{ }^{M}$ expressed in TVE \%. The two bottom graphs refer to the phase of the above-mentioned quantities.

In such a condition, usual TVE values (i.e., 0.1 to $1 \%[4]$ ) lead to major errors in the estimates of both current magnitude and phase. It is worth pointing out that in the graphs of Fig. 2 reporting the phase accuracies, the curves exhibit a knee in the case of high measurement uncertainty. This is due to the fact that the phase error has an upper bound of $\pi$ radians.

Fig. 3 refers to measurement configuration $b$ in which the current phasors are measured and the voltage phasors are calculated. As in the previous case, only $\underline{E}_{b}{ }^{C}$ is shown since the same results are obtained for $\underline{E}_{e}^{C}$. The voltage estimates, especially concerning the phase, are quite inaccurate for usual TVE values. Fig. 4 refers to the measurement configuration $c$ in which voltage and current phasors at the end of the line are measured and the quantities at the beginning of the line are 
calculated. Since we have observed that the accuracy of the computed magnitude is mainly affected by the uncertainty of the measured magnitude and the same remark holds for the phase, only four graphs are shown. The accuracy of both magnitude and phase of the computed quantities is largely improved with respect to configurations $a$ and $b$ : indeed, a TVE of $0.1 \%$ guarantees error stds in the order of $10^{-2} \%$ for the magnitude and $10^{-1} \mathrm{mrad}$ for the phase. Furthermore, it can be noticed that these accuracy levels do not depend on the operating condition.

\section{EXTENSION TO A COMPLEX DISTRIBUTION SYSTEM}

In this section we extend the conclusions obtained for a simple single-phase transmission line to a three-phase distribution system. To this end, we make reference to the IEEE 13-bus distribution test feeder [19]. The influence of different configurations of voltage and/or current synchrophasor measurements is analyzed with respect to the accuracy of a state estimator composed of the DKF proposed in [11].

\section{A. Description of the test feeder and assessment procedure}

The IEEE 13-bus distribution test feeder has been modified as follows: it has a $15 \mathrm{kV}$ rated voltage and the lines, unbalanced, correspond to the configuration \#602 of [19]. Bus \#650 represents the connection to a sub-transmission network characterized by a short circuit power $S_{s c}=300 \mathrm{MVA}$ and a ratio between real and imaginary parts of the short circuit impedance $R_{s c} / X_{s c}=0.1$. The two lines connecting bus \#633 to \#634 and \#671 to \#692 are assumed to be 300 feet long. A load is connected to every bus and two steady-state operating conditions are considered: (i) Case 1 (low-load scenario): each load absorbs $10 \mathrm{kVA}$; (ii) Case 2 (high-load scenario): each load absorbs $1000 \mathrm{kVA}$. In both cases the power is equally distributed among the three phases and a power factor of 0.9 is assumed.

The non-negligible level of imbalances of line parameters and load powers in distribution systems requires the adoption of a three-phase state estimator. The system state is represented by the real and imaginary parts of the voltage phasors in every bus and every phase. The measurements considered in this paper are only voltage and injected current ${ }^{2}$ synchrophasors provided by PMUs. As already stated in the introduction, this hypothesis allows formulating the SE problem in a linear way. Therefore, the measurement set $\mathbf{z}$ is:

$$
\mathbf{z}=\mathbf{H} \mathbf{x}+\mathbf{v},
$$

where matrix $\mathbf{H}$ represents the exact link between measurements and states for the case of null measurement noise, and $\mathbf{v}$ is the measurement noise assumed to be white and Gaussian with covariance matrix $\mathbf{R}$.

As known, the DKF is a two-step estimation process that in the first stage uses a process model, together with the associated covariance matrix $\mathbf{Q}$, to compute the predicted state $\mathbf{x}^{-}$and its covariance $\mathbf{P}^{-}$. Then, it estimates the final state $\mathbf{x}$ and its covariance $\mathbf{P}$ by means of the so-called 'Kalman Gain' $\mathbf{K}$.

\footnotetext{
${ }^{2}$ For the sake of space, current flow synchrophasor measurements are not considered in this paper, however they will be included in a future analysis.
}

In this paper, we adopt the DKF formulation and the $\mathbf{Q}$ assessment method proposed in [11]. The DKF equations are recalled below ( $k$ is the time-step):

- Prediction equations

$$
\begin{gathered}
\mathbf{x}_{k}^{-}=\mathbf{x}_{k-1} \\
\mathbf{P}_{k}^{-}=\mathbf{P}_{k-1}+\mathbf{Q}_{k}
\end{gathered}
$$

- Estimation equations

$$
\begin{gathered}
\mathbf{K}_{k}=\mathbf{P}_{k}^{-} \mathbf{H}^{T}\left(\mathbf{H} \mathbf{P}_{k}^{-} \mathbf{H}^{T}+\mathbf{R}\right)^{-1} \\
\mathbf{x}_{k}=\mathbf{x}_{k}^{-}+\mathbf{K}_{k}\left(\mathbf{z}_{k}-\mathbf{H} \mathbf{x}_{k}^{-}\right) \\
\mathbf{P}_{k}=\left(\mathbf{I}-\mathbf{K}_{k} \mathbf{H}\right) \mathbf{P}_{k}^{-}
\end{gathered}
$$

By assuming to have voltage and/or current synchrophasor measurements in every bus of the system, the DKF state estimator (DKF-SE) accuracy is evaluated for the following measurement configurations:

- Conf. $A$ : voltage phasors in every bus;

- Conf. B: injected current phasors in the slack-bus (bus \#650) and voltage phasors in the other buses;

- Conf. $C$ : injected current phasors in every bus;

- Conf. D: voltage phasors in the slack-bus (bus \#650) and injected current phasors in the other buses;

- Conf. E: voltage and injected current phasors in every bus.

For each scenario, the procedure used to assess the accuracy of the DKF-SE is the following:

1. A power flow is performed to determine the true state of the network;

2. $\quad N$ sets of measurements (as in Section II, we generate $10^{4}$ events) are obtained by perturbing the measured quantities inferred in step 1 with randomly-generated Gaussian noise according to (7). The maximum errors $e r r_{m}$ and $e r r_{p}$ of (7) refer to the cumulated error of a PMU and a 0.1-class sensor. Assuming the sensor error is predominant yields: $\operatorname{err}_{m}=0.1 \%$ and $\operatorname{err}_{p}=1.5 \mathrm{mrad}$ $[20],[21]^{3}$. The corresponding TVE is equal to $0.18 \%$;

3. Each set of measurements is then processed by the DKF$\mathrm{SE}$ in order to get $N$ sets of estimated states. We calculate the estimation errors as the difference between estimated and true state;

4. The SE accuracy is represented by the means and stds of the probability distributions of the estimation errors calculated in step 3 .

\section{B. Numerical example}

This section presents the numerical assessment of the DKF-SE accuracy with respect to the two operating conditions (Case 1 and 2) and the five measurement configurations (Conf. 1-5) described in Sub-Section III.A. As in Section II,

\footnotetext{
${ }^{3}$ In the study carried out in this section, the measurement uncertainties are kept constant since a thorough analysis is beyond the allocated space.
} 
we show the estimation errors of the voltages and injected currents in terms of magnitude and phase. In what follows, when we refer to currents we intend injected ones.
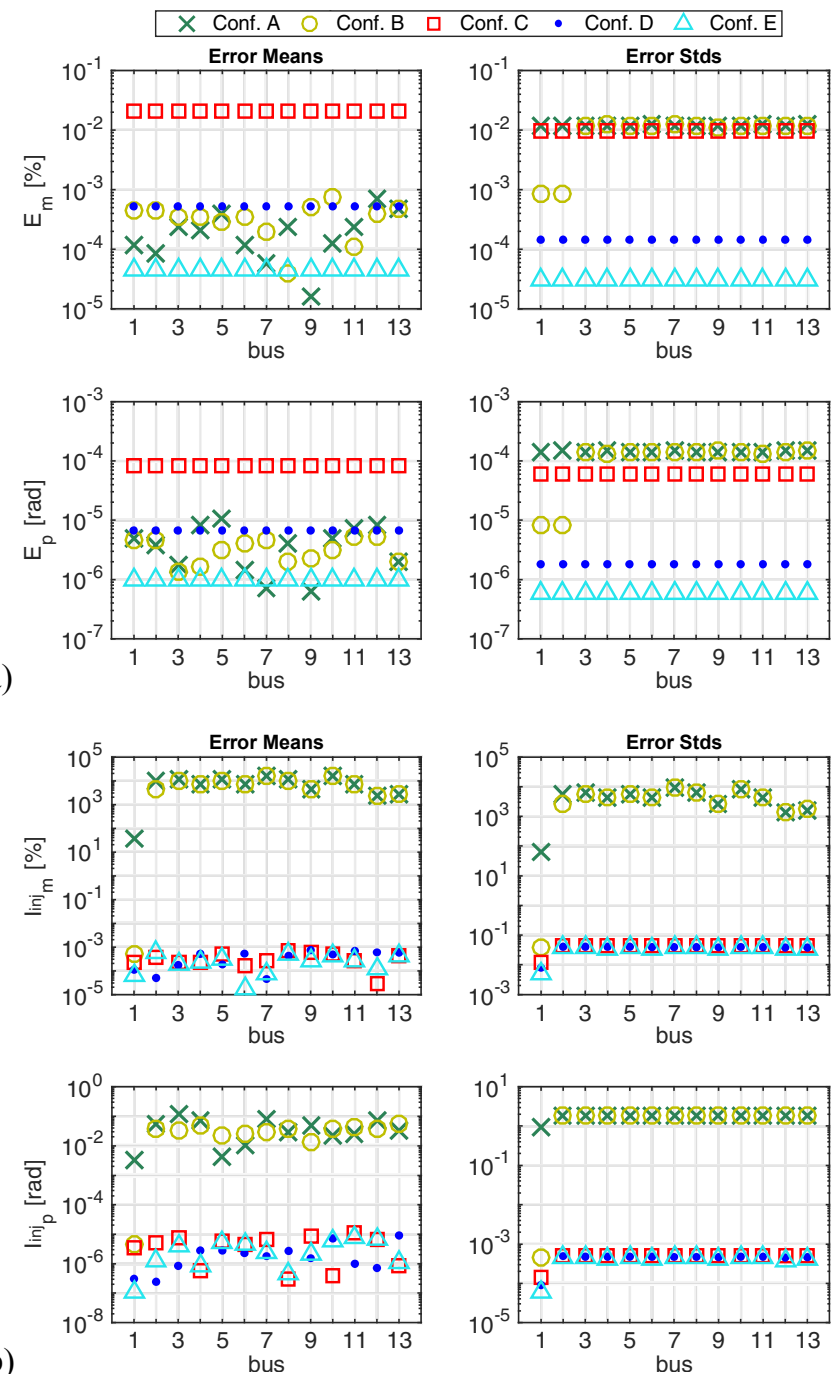

Fig. 5. Case 1. Means and stds of the estimation errors in terms of magnitude and phase of voltages (a) and injected currents (b). For every bus, we consider only the largest error among the three phases. The graphs show the influence on the SE accuracy of the five measurement configurations.

Before commenting on the results, it is worth observing that Conf. $A, B, C$ and $D$ use the same number of measurements. Fig. 5 shows the means and stds of the estimation errors with respect to each bus (only the largest error among the three phases is shown) for Case 1 in which the loads absorb the lowest selected powers. Conf. $A$ and $B$ estimates the voltages with good accuracy, whereas the current estimates are extremely inaccurate in terms of both error means and stds. On the contrary, Conf. $C$ provides accurate current estimates and the worst voltage estimates. Conf. D, consisting of a voltage and (n-1) current measurements, leads to accurate estimates of both voltages and currents in every bus. Finally, the use of all the measurements available (Conf. E) improves only the voltage estimates.
Fig. 6 presents the simulation results for Case 2 referring to the high-load condition. This operating condition is characterized by larger voltage magnitude variations and phase displacements. Indeed, Conf. $A$ and $B$ perform better in terms of current estimates, while Conf. $C$ provides worse voltage estimates with respect to Case 1. Instead, Conf. D and $E$ give again accurate estimates of all the quantities. Note that the same conclusions reported all above can be derived by considering cables instead of overhead lines, power factors different from 0.9 , and unbalanced load powers.
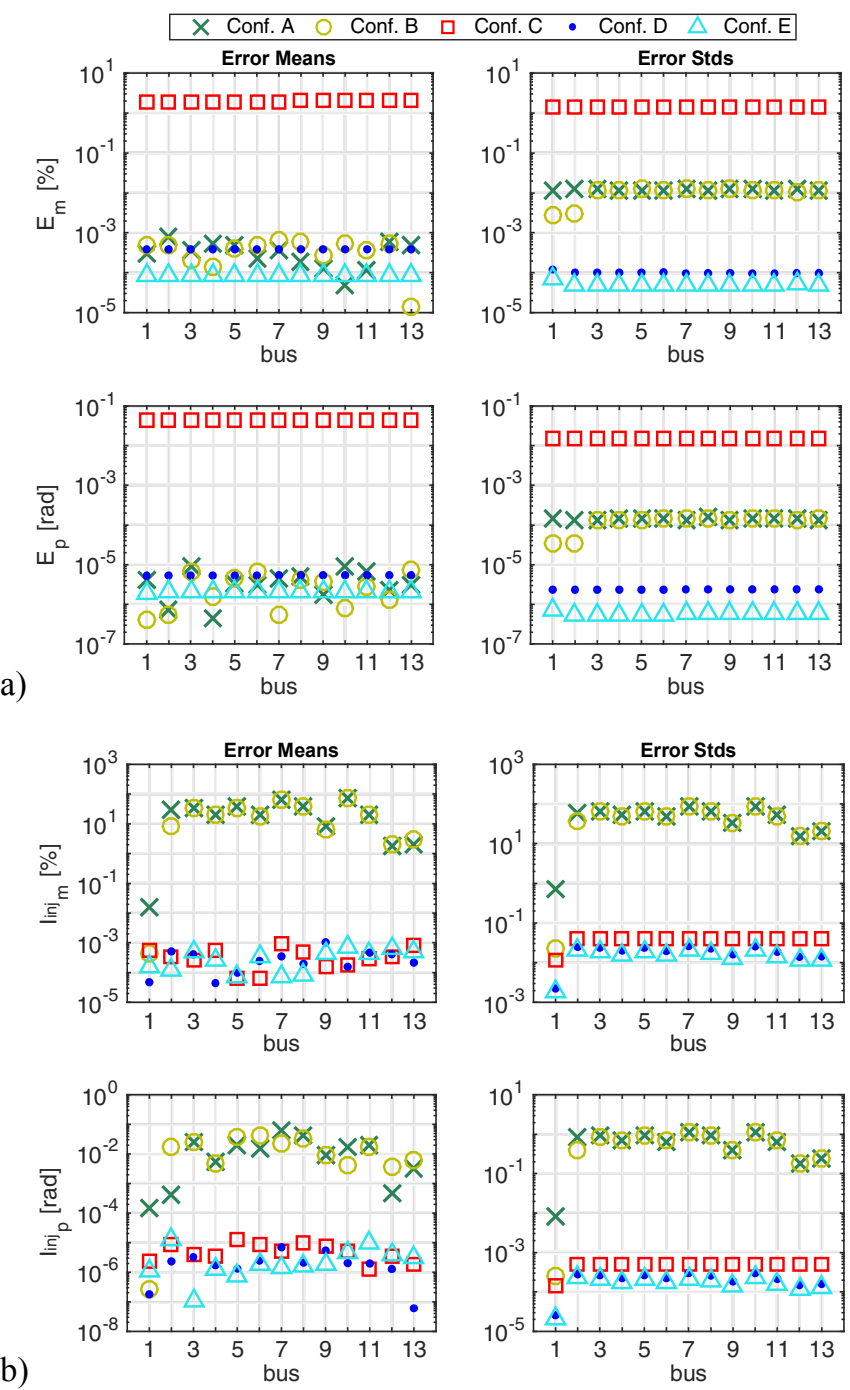

Fig. 6. Case 2. Means and stds of the estimation errors in terms of magnitude and phase of voltages (a) and injected currents (b). For every bus, we consider only the largest error among the three phases. The graphs show the influence on the SE accuracy of the five measurement configurations.

We can observe that measurement configurations composed of only voltages or only currents (Conf. $A$ and $C$ ) are not able to provide accurate estimates in terms of currents and voltages, respectively. A better estimation accuracy is achieved by using mixed voltage and current measurements. The above-reported observations are in agreement with the ones presented in Section II. Moreover, the analysis performed on an entire distribution system allows further investigating the performance of the mixed measurement configurations. In 
particular, it quantifies the different influence of voltage and current measurements on the SE accuracy: Conf. B, consisting mainly on voltage measurements, leads to major errors of the current estimates, whilst Conf. D, composed mainly of current measurements, provides accurate voltage and current estimates. Conf. $E$ improves only the voltage estimates compared to Conf. D.

Hence, we can state that current measurements are crucial for a distribution system linear state estimator. This is due to the specific characteristics and operational conditions of distribution systems resulting into reduced voltage magnitude variations and phase displacements that can be comparable with the uncertainties of the voltage phasor measurements. As a consequence, measurement sets composed mainly of voltages (Conf. $A$ and $B$ ) result in large errors of the current estimates. For example, Conf. $A$ requires measurement uncertainties in the order of $10^{-6} \%$ and $10^{-8} \mathrm{rad}$ in order to get the same SE accuracy of Conf. D. Note that such a phase accuracy is well below the limit of currently available time synchronization systems.

As a conclusion, Conf. $D$ allows obtaining accurate voltage and current estimates irrespectively of the network operating condition and with the minimum number of measurements. The voltage and current estimation errors are always below $0.04 \%$ in terms of magnitude and $0.5 \mathrm{mrad}$ in terms of phase. This accuracy is sufficient for a distribution system operator to exploit most of the functionalities that can be associated to a SE process, such as voltage control, line congestion management, optimal dispatch of DERs.

\section{CONCLUSIONS}

Within the context of distribution systems, the paper has investigated the impact of different combinations of voltage and injected current synchrophasor measurements and their uncertainties on the accuracy of linear SE processes. We have first considered a generic transmission line with characteristic parameters of distribution systems. Using voltage-only or current-only measurements (to compute respectively currents or voltages), we have obtained unacceptable accuracies of the inferred quantities if typical TVE values of PMUs are considered. The best measurement set should include a combination of voltage and current phasors. Then, in order to verify the correctness of these preliminary conclusions for a complex distribution system, we have tested various measurement configurations, together with different operating conditions, by making reference to the IEEE 13-bus test feeder. A DKF-SE fed with voltage and injected current synchrophasors has been adopted. We have analyzed the probability distributions of the voltage and current estimation errors. The mixed measurement configuration composed of voltages in the slack-bus and injected currents in the other buses has achieved the best SE accuracy. In particular, this study has quantified the crucial importance of current phasor measurements to improve the accuracy of distribution system linear state estimators.

\section{REFERENCES}

[1] A. G. Phadke and J. S. Thorp, Synchronized phasor measurements and their applications. New York, NY, USA: Springer, 2008.

[2] S. Sarri, M. Paolone, R. Cherkaoui, A. Borghetti, F. Napolitano, C.A. Nucci, "State estimation of active distribution networks: comparison between WLS and iterated Kalman-filter algorithm integrating PMUs," in 3rd IEEE PES Int. Conf. and Exhib. Innovative Smart Grid Technologies (ISGT Europe), pp. 1-8, 2012.

[3] D. A. Haughton and G. T. Heydt, "A linear state estimation formulation for smart distribution systems," IEEE Trans. Power Syst., vol. 28, no. 2, pp. 1187-1195, May 2013

[4] IEEE Standard for Synchrophasor Measurements for Power Systems, IEEE Standard C37.118.1, 2011.

[5] A. Carta, N. Locci, C. Muscas, S. Sulis, "A Flexible GPS-Based System for Synchronized Phasor Measurement in Electric Distribution Networks", IEEE Trans. Instrum. Meas., vol. 57, no. 11, pp. 24502456, Nov. 2008.

[6] P. Romano, M. Paolone, "Enhanced Interpolated-DFT for Synchrophasor Estimation in FPGAs: Theory, Implementation, and Validation of a PMU Prototype," IEEE Trans. Instrum. Meas., vol. 63, no. 12, pp. 2824-2836, Dec. 2014.

[7] P. Romano, M. Paolone, "An enhanced interpolated-modulated DFT for high reporting rate PMUs," in IEEE Int. Workshop Applied Measurements for Power Systems (AMPS), pp. 1-6, 2014.

[8] C.N. Lu, J.H. Teng, W.-H.E. Liu, "Distribution system state estimation," IEEE Trans. Power Syst., vol.10, no.1, pp. 229-240, Febr. 1995.

[9] M. E. Baran, and A. W. Kelley, "A branch-current-based state estimation method for distribution systems," IEEE Trans. Power Syst., vol. 10, no. 1, pp. 483-491, Febr. 1995.

[10] K. D. Jones, J. S. Thorp, and R. M. Gardner, "Three-phase linear state estimation with phasor measurements," in IEEE PES General Meeting, pp. 1-5, 2013.

[11] L. Zanni, S. Sarri, M. Pignati, R. Cherkaoui, and M. Paolone, "Probabilistic assessment of the process-noise covariance matrix of discrete Kalman filter state estimation of active distribution networks," presented at the $201410^{\text {th }}$ Int. Conf. Probabilistic Methods Applied to Power Systems (PMAPS), Durham, UK, July 7-10, 2014.

[12] N. M. Manousakis, G. N. Korres, and P. S. Georgilakis, "Taxonomy of PMU placement methodologies," IEEE Trans. Power Syst., vol. 27, no. 2, pp. 1070-1077, May 2012.

[13] K. Li, "State estimation for power distribution system and measurement impacts," IEEE Trans. Power Syst., vol. 11, no. 2, pp. 911-916, May 1996.

[14] R. Singh, B.C. Pal, R.B. Vinter, "Measurement Placement in Distribution System State Estimation," IEEE Trans. Power Syst., vol. 24, no. 2, pp. 668-675, May 2009.

[15] E. Caro, R. Singh, B.C. Pal, A.J. Conejo, and R.A. Jabr, "Participation factor approach for phasor measurement unit placement in power system state estimation," IET Gener., Transm., Distrib., vol. 6, no. 9, pp. 922-929, 2012.

[16] J. Liu, J. Tang, F. Ponci, A. Monti, C. Muscas, P.A. Pegoraro, "TradeOffs in PMU Deployment for State Estimation in Active Distribution Grids," IEEE Trans. Smart Grid, vol. 3, no. 2, pp. 915-924, June 2012.

[17] C. Muscas, S. Sulis, A. Angioni, F. Ponci, A. Monti, "Impact of Different Uncertainty Sources on a Three-Phase State Estimator for Distribution Networks," IEEE Trans. Instrum. Meas., vol. 63, no. 9, pp. 2200-2209, Sept. 2014.

[18] BIPM, IEC, IFCC, ILAC, IUPAC, IUPAP, ISO, OIML (2008) Evaluation of measurement data - guide for the expression of uncertainty in measurement. JCGM 100: 2008. http://www.bipm.org/ en/publications/guides/gum.html

[19] IEEE Distribution Planning Working Group, "Radial distribution test feeders," IEEE Trans. on Power Syst., vol. 6, pp. 975-985, Aug. 1991.

[20] Standard, I.E.C. 60044-1, "Instrument transformers Part 1: Current voltage transformers," Geneva (Switzerland), 1996.

[21] Standard, I.E.C. 60044-2, "Instrument transformers Part 2: Inductive voltage transformers," Geneva (Switzerland), 1997. 